\title{
Regional differences: clinical practice guidelines on the management of hepatocellular carcinoma
}

\author{
Yuquan Qian ${ }^{1}$, Andreas Teufel ${ }^{1,2}$ \\ ${ }^{1}$ Division of Hepatology, Department of Medicine II, Medical Faculty Mannheim, University of Heidelberg, Mannheim, Germany; ${ }^{2}$ Clinical \\ Cooperation Unit Healthy Metabolism, Center for Preventive Medicine and Digital Health Baden-Württemberg (CPDBW), Medical Faculty \\ Mannheim, University of Heidelberg, Mannheim, Germany \\ Correspondence to: Prof. Dr. Dr. Andreas Teufel. Professor of Hepatology, Division of Hepatology, Division of Bioinformatics, Department of \\ Medicine II, Medical Faculty Mannheim, University of Heidelberg, 68167 Mannheim, Germany. Email: andreas.teufel@medma.uni-heidelberg.de. \\ Comment on: Xie DY, Ren ZG, Zhou J, et al. 2019 Chinese clinical guidelines for the management of hepatocellular carcinoma: updates and insights. \\ Hepatobiliary Surg Nutr 2020;9:452-63.
}

Submitted Nov 28, 2021. Accepted for publication Dec 06, 2021.

doi: 10.21037/hbsn-21-496

View this article at: https://dx.doi.org/10.21037/hbsn-21-496

Hepatocellular carcinoma (HCC) remains a global health burden, stimulating the scientific community to further deepen their research in this field. In order to successfully translate novel insights into clinical practice and provide clinicians with evidence-based care for their patients, HCC clinical practice guidelines were published and need to be updated periodically.

China has a plethora of patients who suffer from HCC. However, in contrast to Western countries, the underlying disease in Chinese HCC patients is mainly hepatitis B. This considerable difference may well influence diagnostics and efficacy of treatments, particularly systemic therapy. Thus, it is reasonable for the Chinese hepatology community to update their HCC clinical guidelines independently from Western hepatology societies such as EASL or AASLD. However, their comparison is certainly stimulating to the scientific discussion. Therefore, Xie et al. just published such a nice comparison of the latest update of the Chinese guidelines published in 2019 to recommendations from EASL and AASLD (1).

While sharing many similarities, the etiological and thus molecular differences lead to clearly distinct recommendation for the use of the tumor marker AFP for HCC surveillance and may also influence selection of systemic therapy options.

In contrast to EASL not recommending AFP for surveillance due to false positive screening and costeffectiveness calculations, the Chinese guidelines recommend AFP. Xie et al. point out that HBV-related HCCs tend to have higher AFP levels compared to HCC due to other causes (1). Although this may be the case, solid data supporting AFP efficacy particularly in HBVrelated HCC is lacking. The reference to the work by Tayob et al. retrospectively investigating AFP sensitivity in the HALT-C trial population certainly need further confirmation as these patients suffered from hepatitis $\mathrm{C}$ (2). Just recently novel trials investigating AFP, AFP-L3, DCP and the GALAD score $(3,4)$ as the most prominent tumor markers for HCC were reported to perform unsatisfactory at the AASLD annual meeting. The GALAD score, which performed best in terms of detection rate was demonstrated to be associated with a high false positive prediction rate of over $20 \%$ (5). Thus, the debate about the use of AFP and other biomarkers for surveillance and early detection of HCC remains challenging and may not only be a matter of different etiologies or ethnic background among Chinese and Western patients.

Furthermore, the landscape of systemic therapy has significantly changed over the past two years. With the results from the IMbrave150 trial being reported at 2019 ESMO Asia, systemic treatment of HCC has entered a new level. Combination of atezolizumab and bevacizumab yielded a significantly improved overall survival in comparison to sorafenib treatment (6). However, subgroup analysis demonstrated best efficacy of this immunotherapy in patients with viral hepatitis, which may be particularly 
interesting in China. With additional companies releasing press notes on the efficacy of durvalumab and tremelimumab in the HIMALAYA phase III trial (7) and atezolizumab and cabozantinib in COSMIC-312 phase III trial (8) we are certainly at the beginning of a new era. Therefore the future development of the Chinese PD-1 inhibitor camrelizumab will also be of great interest. Given these recent developments, the role of TKIs such as lenvatinib or sorafenib will have to be re-defined, just as the role of former second-line therapeutics, such as cabozantinib, ramucirumab, or regorafenib.

Overall, many common paradigms regarding surveillance, diagnosis, and treatment are shared among the Chinese and Western recommendations. However, a few differences remain. Given the etiological and ethnic differences, joint efforts and clinical trials would be extremely valuable to further improve HCC diagnostics and treatment. The elaborate summary by Xie et al. is certainly a valuable initiative to foster joint efforts to fight HCC.

\section{Acknowledgments}

Funding: Prof. AT was supported by the Sino-German Center for Research Promotion (GZ-1546 and C-0012), the State Ministry of Baden-Wuerttemberg for Sciences, Research and Arts supporting the Clinical Cooperation Unit Healthy Metabolism at the Center for Preventive Medicine and Digital Health (CCU Healthy Metabolism), and the Baden-Wuerttemberg Center for Digital Early Disease Detection and Prevention (BW-ZDFP).

\section{Footnote}

Provenance and Peer Review: This article was commissioned by the editorial office, Hepatobiliary Surgery and Nutrition. The article did not undergo external peer review.

Conflicts of Interest: Both authors have completed the ICMJE uniform disclosure form (available at https://hbsn. amegroups.com/article/view/10.21037/hbsn-21-496/coif). AT reports grants from the Sino-German Center for Research Promotion (GZ-1546 and C-0012), the State Ministry of Baden-Wuerttemberg for Sciences, Research and Arts supporting the Clinical Cooperation Unit Healthy Metabolism at the Center for Preventive Medicine and Digital Health (CCU Healthy Metabolism), and the BadenWuerttemberg Center for Digital Early Disease Detection and Prevention (BW-ZDFP). He also reports receiving consulting fees from Ipsen Pharma GmbH, Gilead Sciences, AbbVie Deutschland GmbH \& Co. KG, F. HoffmannLa Roche AG, Eisai GmbH, Bayer AG, Novartis AG, Intercept Pharmaceuticals, Inc., Lilly Deutschland GmbH and payments or honoraria for lectures from Ipsen Pharma GmbH, Gilead Sciences, AbbVie Deutschland GmbH \& Co. KG, F. Hoffmann-La Roche AG, Eisai GmbH, Bayer AG, Novartis AG, Intercept Pharmaceuticals, Inc., Lilly Deutschland GmbH, esanum GmbH, Mediverbund AG, RG Gesellschaft fuer Information und Organisation mbH. The other author has no conflicts of interest to declare.

Ethical Statement: The authors are accountable for all aspects of the work in ensuring that questions related to the accuracy or integrity of any part of the work are appropriately investigated and resolved.

Open Access Statement: This is an Open Access article distributed in accordance with the Creative Commons Attribution-NonCommercial-NoDerivs 4.0 International License (CC BY-NC-ND 4.0), which permits the noncommercial replication and distribution of the article with the strict proviso that no changes or edits are made and the original work is properly cited (including links to both the formal publication through the relevant DOI and the license). See: https://creativecommons.org/licenses/by-nc-nd/4.0/.

\section{References}

1. Xie DY, Ren ZG, Zhou J, et al. 2019 Chinese clinical guidelines for the management of hepatocellular carcinoma: updates and insights. Hepatobiliary Surg Nutr 2020;9:452-63.

2. Tayob N, Lok AS, Do KA, et al. Improved Detection of Hepatocellular Carcinoma by Using a Longitudinal AlphaFetoprotein Screening Algorithm. Clin Gastroenterol Hepatol 2016;14:469-475.e2.

3. Caviglia GP, Abate ML, Petrini E, et al. Highly sensitive alpha-fetoprotein, Lens culinaris agglutininreactive fraction of alpha-fetoprotein and des-gammacarboxyprothrombin for hepatocellular carcinoma detection. Hepatol Res 2016;46:E130-5.

4. Best J, Bechmann LP, Sowa JP, et al. GALAD Score Detects Early Hepatocellular Carcinoma in an International Cohort of Patients With Nonalcoholic Steatohepatitis. Clin Gastroenterol Hepatol 2020;18:728735.e4.

5. El-Serag HB, Tayob N, Kanwal F, et al. The performance 
of AFP, AFP-3, DCP as biomarkers for detection of hepatocellular carcinoma (HCC). A phase 3 biomarkers study in the United States. TLMdx Abstract Oral Presentation 1592021.

6. Finn RS, Qin S, Ikeda M, et al. Atezolizumab plus Bevacizumab in Unresectable Hepatocellular Carcinoma. N Engl J Med 2020;382:1894-905.

7. AstraZeneca. Imfinzi plus tremelimumab significantly improved overall survival in HIMALAYA Phase III trial in 1st-line unresectable liver cancer. 2021. Available online: https://www.astrazeneca.com/media-centre/pressreleases/2021/imfinzi-and-tremelimumab-improved-os-

Cite this article as: Qian Y, Teufel A. Regional differences: clinical practice guidelines on the management of hepatocellular carcinoma. HepatoBiliary Surg Nutr 2022;11(1):161-163. doi: 10.21037/hbsn-21-496 in-liver-cancer.html

8. Ipsen. Exelixis and Ipsen Announce Cabozantinib in Combination with an Immune Checkpoint Inhibitor Significantly Improved Progression-Free Survival in Phase 3 COSMIC-312 Pivotal Trial in Patients with Previously Untreated Advanced Liver Cancer. 2021. Available online: https://www.ipsen.com/press-releases/exelixis-and-ipsenannounce-cabozantinib-in-combination-with-an-immunecheckpoint-inhibitor-significantly-improved-progressionfree-survival-in-phase-3-cosmic-312-pivotal-trial-inpatients-with-previo/ 